\title{
VECTOR LATTICES WITH DUALS OF INTEGRAL TYPE
}

\author{
H. W. ELLIS*
}

1. Introduction. Marston Morse and William Transue $(\mathbf{4} ; \mathbf{5} ; \mathbf{6})$ have developed a theory of seminormed Banach spaces of complex or real-valued functions called MT spaces. For $E$ a locally compact space, $\Re^{E}$ the space of real-valued functions on $E$, and $\mathrm{K}$ the space of real-valued functions on $E$ with compact supports, a real MT space $A$ is a vector subspace of $\Re^{E}$ containing $\mathrm{K}$ and provided with a suitable seminorm $N^{A}$. Each element $\phi$ of the $N^{A}$-dual $A^{*}$ of $A$ determines a Radon measure $\mu_{\varphi}$ on $E$, and $\phi(f)=\int f d \mu_{\varphi}$ is valid on $A$ where the integration is in the Bourbaki sense. Problems considered include the compatibility of different MT spaces and the relations between the MT spaces and the spaces $L_{1}(\phi)$ of integrals determined by the measure dual.

In this paper $\Re^{E}$ is replaced by an arbitrary $\sigma$-Dedekind complete space $R$ and $\mathrm{K}$ by a Riesz subspace $C$ of $R$. Certain order-bounded linear functionals $\phi$ on $C$, called integrals, replace the Radon measure functionals and determine integration spaces $L_{1}(\phi)$ by an extension method of Nakano (7). If $A$ is a Riesz subspace of $R$ containing $C$ and $N^{A}$ is a suitable seminorm on $A$, every element $\phi$ of $A^{*}$ determines an integral on $C$ for which $\phi$ is the Nakano extension on $A$. Such a pair $\left(A, N^{A}\right)$ is called an abstract Morse-Transue or AMT space. The principal MT results extend to the abstract case, the arguments now being lattice theoretic. Recent work of Luxemburg and Zaanen (3), in part drawing on earlier work by Nakano, helps to clarify and extend the theory ( $\$ 5$ below). The theory of MT spaces and part of the theory of $L^{\lambda}$ spaces (2) is included in the AMT theory for suitable choices of $R$ and $C$.

The author wishes to express his appreciation to Professor Hidegoro Nakano for suggesting the topic of this investigation and for invaluable suggestions concerning the vector lattice setting.

2. Integrals and their Nakano extensions. We shall use in general the definitions of (3). A vector or linear lattice $R$ is $\sigma$-Dedekind complete (sequentially continuous) if $a_{n} \in R^{+}, n=1,2, \ldots$ implies that $\wedge_{1}^{\infty} a_{n} \in R$; Dedekind complete (universally continuous) if for every collection $a_{\lambda} \in R^{+}, \lambda \in \Lambda$, $\wedge_{\lambda \in \Lambda} a_{\lambda} \in R$; super Dedekind complete (super-universally continuous) if it is Dedekind complete and such that there exists a countable subsequence $a_{\lambda i}$, $\lambda_{i} \in \Lambda$, with $\wedge_{i} a_{\lambda i}=\wedge_{\lambda \in \Lambda} a_{\lambda}$. A linear subspace of a linear lattice is called a Riesz subspace if it is closed under the lattice operations. A Riesz subspace of a $\sigma$-Dedekind complete vector lattice $R$ is called a sequentially continuous or

Received June 14, 1966.

*Partly supported by a National Research Council (Canada) Senior Research Fellowship. 
$\sigma$-Dedekind complete subspace if it is a $\sigma$-Dedekind complete vector space contained in $R$ that is closed under countable infima and suprema. A Riesz subspace $S$ of $R$ is an ideal if $x \in S, a \in R$ with $|a| \leqslant|x|$ implies that $a \in S$.

Let $C$ be a Riesz subspace of a $\sigma$-Dedekind complete vector lattice $R, C^{\sim}$ the set of order-bounded linear functionals on $C$. If $u_{n} \in C^{+}, n=1,2, \ldots, \wedge_{n=1}^{\infty} u_{n}$ exists in $R^{+}$, the elements being considered as elements of $R$. If $u_{n} \downarrow$ and $\wedge u_{n}=0$ in $R$, we write $u_{n} \downarrow 0(R)$. Observe that if $C$ is not sequentially continuous, the collection $\left\{u_{n}\right\}$ need not have an infimum in $C$ or may have an infimum different from that in $R$.

Let $C_{c}^{\sim}=\left(\phi \in C^{\sim}: u_{n} \downarrow 0\right.$ implies $\phi\left(u_{n}\right) \rightarrow 0$ as $\left.n \rightarrow \infty\right)(3, \mathrm{VI}) ; C_{\bar{c}}^{\sim}=(\phi$ $\in C^{\sim}: u_{n} \downarrow 0(R)$ implies that $\phi\left(u_{n}\right) \rightarrow 0$ as $\left.n \rightarrow \infty\right)$. Always $C_{\bar{c}}^{\sim} \supset C_{c}^{\sim} \supset\{0\}$. It is shown in (3, VII, p. 674) that for $C=C[0,1]$, the continuous functions on $[0,1], C_{c} \sim=\{0\}$. For $E$ a locally compact space, $C$ the continuous functions on $E$ with compact supports, $R=\Re^{E}, u_{n} \downarrow 0(R)$ implies that $u_{n} \rightarrow 0$ uniformly and this implies that $C_{\bar{c}}^{\sim}=C^{\sim}$. Here again $C_{c}^{\sim}$ may reduce to $\{0\}$. The elements of $C_{\bar{c}}^{\sim}$ will be called $C$-integrals or just integrals. When $C$ is a sequentially continuous Riesz subspace of $R, C_{\bar{c}}^{\sim}=C_{c}^{\sim}$. The spaces $C_{c}^{\sim}$ and $C_{\bar{c}}^{\sim}$ are normal subspaces of the universally continuous linear lattice $C^{\sim}$ ordered pointwise on $C$, the arguments for $C_{\bar{c}} \sim$ being analogous to those for $C_{c}^{\sim}(3, \mathrm{VI})$.

Using the method of Nakano (7) we extend each positive $\phi \in C_{\bar{c}}^{\sim}$ to upper and lower functionals on $R$. For each $x \in R$ we set

$$
\begin{aligned}
\phi^{*}(x) & =\inf \left(\lim _{i \rightarrow \infty} \phi\left(a_{i}\right), \text { for all increasing sequences }\left(a_{i} \uparrow\right), a_{i} \in C,\right. \\
& \left.=+\infty \text { if no such sequence exists; } \quad \text { with } \vee\left(x \wedge a_{i}\right)=x(\text { in } R)\right) \\
\phi_{*}(x) & =\sup \left(\lim _{i \rightarrow \infty} \phi\left(b_{i}\right), \text { for all sequences } b_{i \downarrow}, b_{i} \in C,\right. \\
& \left.=-\infty, \text { if no such sequence exists. } \quad \text { with } \wedge\left(x \vee b_{i}\right)=x(\text { in } R)\right)
\end{aligned}
$$

As shown in (7) the functionals $\phi^{*}$ and $\phi *$ have the following properties (with values in the extended reals):

N1. $\phi^{*}(0)=\phi_{*}(0)=0$.

$\mathrm{N} 2 . \phi^{*}(-x)=-\phi_{*}(x), \phi_{*}(x)=-\phi^{*}(x)$.

N3. $\phi^{*}(a x)=a \phi^{*}(x), \phi_{*}(a x)=a \phi_{*}(x)$ if $a \geqslant 0$.

N4. $\phi^{*}(x)+\phi^{*}(y) \geqslant \phi^{*}(x+y) \geqslant \phi^{*}(x)+\phi *(y)$,

when the sums have sense. Similarly

$$
\phi_{*}(x)+\phi_{*}(y) \leqslant \phi_{*}(x+y) \leqslant \phi^{*}(x)+\phi_{*}(y) .
$$

N5. $\phi^{*}(x)+\phi^{*}(y) \geqslant \phi^{*}(x \vee y)+\phi^{*}(x \wedge y)$,

$$
\phi_{*}(x)+\phi_{*}(y) \leqslant \phi_{*}(x \vee y)+\phi_{*}(x \wedge y) \text {. }
$$

N6. $\phi^{*}(x) \geqslant \phi_{*}(x)$.

N7. $\phi^{*}(x) \geqslant \phi^{*}(y), \phi_{*}(x) \geqslant \phi_{*}(y)$ if $x \geqslant y$. 
N8. $\lim \phi^{*}\left(x_{n}\right)=\phi^{*}(x)$ if $x_{n} \uparrow x$.

N9. $\lim \phi_{*}\left(x_{n}\right)=\phi_{*}(x)$ if $x_{n} \downarrow x$.

N10. $\phi^{*}(a)=\phi_{*}(a)=\phi(a)$ for all $a \in C$.

We note the following additional properties:

2.1. If $x_{n} \uparrow x\left(x_{n} \downarrow x\right), x_{n} \in C, x \in R$, then $\phi^{*}(x)=\phi_{*}(x)$.

If $x_{n} \uparrow x, \phi_{*}(x) \geqslant \phi\left(x_{n}\right), n=1,2$, by N7 whence

$$
\phi_{*}(x) \geqslant \lim _{n \rightarrow \infty} \phi\left(x_{n}\right)=\phi^{*}(x),
$$

by N8. With N6 this gives the first part of 2.1.

2.2. From $\mathrm{N} 4$ and $\mathrm{N} 10$, if $x \in R, u \in C$,

$$
\phi^{*}(x+u)=\phi^{*}(x)+\phi(u), \quad \phi_{*}(x+u)=\phi_{*}(x)+\phi(u) .
$$

2.3. From 2.2 and $\mathrm{N} 2$, for all $u \in C$,

$$
\begin{aligned}
\phi^{*}(x)-\phi_{*}(x) & =\phi^{*}(x+u)-\phi_{*}(x+u) \\
& =\phi^{*}(x-u)+\phi^{*}(u-x) .
\end{aligned}
$$

For $\varphi \geqslant 0$ let $L_{1}(\phi)$ denote the elements $x \in R$ for which $\phi^{*}(x)=\phi_{*}(x)$, the common value being finite. Then $L_{1}(\phi)$ is a sequentially continuous Riesz subspace of $R$ containing $C$. Setting $\phi=\phi^{*}=\phi_{*}$ on $L_{1}(\phi), \phi$ is linear on $L_{1}(\phi)$.

Lемма 2.1. Let $L$ be an arbitrary Riesz space and suppose that $u_{n} \wedge f \uparrow f$, $v_{n} \wedge f \uparrow f$. Then if $w_{n}=u_{n} \wedge v_{n}, w_{n} \wedge f \uparrow f$.

Proof. Clearly $f \wedge w_{n} \leqslant f, n=1,2, \ldots$ Let $h$ be an arbitrary upper bound of this set. Then

$$
\begin{aligned}
h & \geqslant u_{m} \wedge v_{m} \wedge f \geqslant u_{n} \wedge v_{m} \wedge f, & & n \text { fixed, } m>n, \\
& \geqslant u_{n} \wedge f, & & n=1,2, \ldots .
\end{aligned}
$$

Thus $h \geqslant f$ and $f=\vee\left(w_{n} \wedge f\right)$.

TheOREM 2.1. (i) If $\phi \in C_{\bar{c}} \sim$ and $a \geqslant 0$, then $a \phi^{*}=(a \phi)^{*}$ and $a \phi_{*}=(a \phi)_{*}$ on R. (ii) If $\phi_{1}, \phi_{2} \in C_{\bar{c}}^{\tilde{c}^{+}}$, then $\left(\phi_{1}+\phi_{2}\right)^{*}=\phi_{1}{ }^{*}+\phi_{2}{ }^{*}$ and $\left(\phi_{1}+\phi_{2}\right)_{*}=\phi_{1 *}$ $+\phi_{2 *}$ on $R^{+}$. (iii) If $\phi_{1} \geqslant \phi_{2} \geqslant 0$ on $C^{+}, \phi_{1}{ }^{*} \geqslant \phi_{2}{ }^{*}$ and $\phi_{1 *} \geqslant \phi_{2 *}$ on $R^{+}$.

Proof of (ii). Let $\phi=\phi_{1}+\phi_{2}$ in $C_{\bar{c}^{-+}}$. Let $\left\{u_{n}\right\}$ be a monotone sequence in $C^{+}$. Then $0 \leqslant \phi\left(u_{n}\right)=\phi_{1}\left(u_{n}\right)+\phi_{2}\left(u_{n}\right)<\infty, n=1,2, \ldots$, and

$$
\lim _{n \rightarrow \infty} \phi\left(u_{n}\right)=\lim _{n \rightarrow \infty} \phi_{1}\left(u_{n}\right)+\lim _{n \rightarrow \infty} \phi_{2}\left(u_{n}\right)
$$

in the non-negative extended reals $\bar{\Re}^{+}$. If $u \in R^{+}$with $\phi_{1}^{*}(u)<\infty$ and $\phi_{2}{ }^{*}(u)<\infty$, there are sequences $u_{n} \uparrow, u_{n} \wedge u \uparrow u$ with the limits on the right simultaneously near $\phi_{1}{ }^{*}(u)$ and $\phi_{2}{ }^{*}(u)$ (using Lemma 2.1). This implies that

$$
\phi^{*}(u) \leqslant \phi_{1}^{*}(u)+\phi_{2}^{*}(u) .
$$


Since $\phi^{*}(u)$ is finite, there exist such sequences with $\lim \phi\left(u_{n}\right)$ arbitrarily near $\phi^{*}(u)$, leading to the opposite inequality and thus to equality in $(*)$. Similar considerations show that if one side is infinite so is the other. Similar arguments using decreasing sequences give the result for the lower extensions.

Theorem 2.2. If $\phi_{1}, \phi_{2} \in C_{\bar{c}}^{-+}$and $\phi=\phi_{1}+\phi_{2}$, then

$$
L_{1}(\phi)=L_{1}\left(\phi_{1}\right) \cap L_{1}\left(\phi_{2}\right) \text {. }
$$

Proof. On $L_{1}\left(\phi_{1}\right) \cap L_{1}\left(\phi_{2}\right), \phi^{+*}=\phi^{+}{ }_{*}, \phi^{-*}=\phi^{-}{ }_{*}$ and all are finite. Thus Theorem 2.1 (ii) shows that $|\phi|^{*}=|\phi|_{*}$ on positive elements. If $u$ is arbitrary, N4 and N2 then imply that

$$
|\phi|^{*}(u)=|\phi|^{*}\left(u^{+}\right)-|\phi|^{*}\left(u^{-}\right)=|\phi|_{*}\left(u^{+}\right)-|\phi|_{*}\left(u^{-}\right)=|\phi|_{*}(u)
$$

and $u \in L_{1}(|\phi|)$.

On $L_{1}(|\phi|)^{+},|\phi|^{*}=|\phi|_{*}$ and both are finite. By Theorem $2.1, \phi^{+*}+\phi^{-*}$ $=|\phi|^{*}=|\phi|_{*}=\phi^{+}{ }_{*}+\phi^{-}$. Since all are positive on positive elements and $\phi^{+*} \geqslant \phi^{+}{ }_{*}, \phi^{-*} \geqslant \phi^{-}$, it follows that $\phi^{+*}=\phi^{+}{ }_{*}$ and $\phi^{-*}=\phi^{-}$. Thus $L_{1}(|\phi|)$ $\subset L_{1}\left(\phi_{1}\right) \cap L_{1}\left(\phi_{2}\right)$ and the proof is complete.

Let $\phi \in C_{\bar{c}}^{-}$. Then $\phi=\phi^{+}-\phi^{-}$on $C$ with $\phi^{+}, \phi^{-} \in C_{\bar{c}}^{-+}$. Writing $|\phi|=\phi^{+}$ $+\phi^{-},|\varphi|, \varphi^{+}$, and $\varphi^{-}$on $C$ have linear extensions $|\phi|=|\phi|^{*}=|\varphi|_{*}, \phi^{+}=\phi^{+*}$ $=\phi^{+}$, and $\phi^{-}=\phi^{-*}=\phi^{-}$to $L_{1}(|\phi|)$. The linear extension of an arbitrary $\phi$ is now defined to be $\phi^{+}-\phi^{-}$on $L_{1}(|\phi|)$ and $L_{1}(\phi)$ is defined to coincide with $L_{1}(|\phi|)=L_{1}\left(\phi^{+}\right) \cap L_{1}\left(\phi^{-}\right)$. For all $x$ in $L_{1}(\phi)$

$\phi(x)=\phi^{+}(x)-\phi^{-}(x)=\phi^{+}\left(x^{+}\right)-\phi^{+}\left(x^{-}\right)-\phi^{-}\left(x^{+}\right)+\phi^{-}\left(x^{-}\right),|\phi(x)| \leqslant|\phi|(|x|) \cdot$

A seminorm $\rho$ on a Riesz space $L$ is called a Riesz or monotone seminorm if $\rho(f) \leqslant \rho(g)$ when $|f| \leqslant|g|$. On $L_{1}(\phi)=L_{1}(|\phi|) \rho \varphi$, defined by $\rho \varphi(u)=|\phi|(|u|)$, is a Riesz seminorm.

ThEOREM 2.3. $C$ is dense in $L_{1}(\phi)$ for the $\rho \varphi$ seminorm topology.

Proof. There is no loss of generality in assuming that $\phi>0$ and $f>0$. Then $\rho \varphi=\phi$ is linear on $L_{1}(\phi)^{+}$. From the definition of $\phi=\phi^{*}$ on $L_{1}(\phi)$, given $\epsilon>0$ there exists a sequence $\left\{u_{n}\right\}$ in $C$ with $u_{n} \uparrow, u_{n} \wedge f \uparrow f$, and

$$
\lim _{n \rightarrow \infty} \phi\left(u_{n}\right) \geqslant \phi(f)=\phi^{*}(f) \geqslant \lim _{n \rightarrow \infty} \phi\left(u_{n}\right)-\epsilon / 4, \quad \phi\left(u_{n} \wedge f\right) \uparrow_{n=1}^{\infty} \phi(f) .
$$

Thus for $n$ sufficiently large

$$
\begin{gathered}
\left|\phi(f)-\phi\left(u_{n}\right)\right|=\left|\phi\left(f-u_{n}\right)\right|<\epsilon / 4 \\
0 \leqslant \phi\left(u_{n}\right)-\phi\left(u_{n} \wedge f\right)=\phi\left(u_{n}-u_{n} \wedge f\right) \leqslant\left|\phi\left(u_{n}-f\right)\right|+\phi\left(f-u_{n} \wedge f\right)<\epsilon / 4 . \\
\text { Now } \quad \begin{array}{c}
0 \leqslant\left|f-u_{n}\right|-\left(f-u_{n}\right)=2\left(f-u_{n}\right)^{-}=2\left(u_{n}-u_{n} \wedge f\right) \\
0 \leqslant \phi\left(\left|f-u_{n}\right|\right)-\phi\left(f-u_{n}\right)=2 \phi\left(u_{n}-u_{n} \wedge f\right)<\epsilon / 2, \\
\phi\left(\left|f-u_{n}\right|\right) \leqslant\left|\phi\left(f-u_{n}\right)\right|+\epsilon / 2<\epsilon .
\end{array}
\end{gathered}
$$


3. Abstract Morse-Transue (AMT) spaces. Fix $R$, a $\sigma$-Dedekind or Dedekind complete Riesz space, and $C$ a Riesz subspace of $R$. Let $A$ be a Riesz subspace of $R$ containing $C$ and suppose that $A$ has a non-trivial Riesz seminorm $N^{A}$.

Let $A^{*}$ denote the dual of $A$ for the seminorm topology, $A_{\bar{c}}^{*}=A^{*} \cap A_{\bar{c}}$. For $\phi \in A^{\sim}$ let $\phi_{c}$ denote the restriction of $\phi$ to $C$. If $\phi \in A_{\bar{c}}^{*}$ and $u_{n} \downarrow 0(R)$ with the elements $u_{n}$ in $C$ and thus in $A, \phi_{c}\left(u_{n}\right)=\phi\left(u_{n}\right) \rightarrow 0$ as $n \rightarrow \infty$ so that $\phi_{c} \in C_{\bar{c}}$. Thus to each $\phi \in A_{\bar{c}}{ }^{*}$ corresponds a space of integrable elements $L_{1}\left(\phi_{c}\right)$. We define

$$
\Omega^{A}=\bigcap_{\varphi \in A_{\bar{c}^{*}}} L_{1}\left(\phi_{c}\right)=\bigcap_{0 \leqslant \varphi \in A_{\bar{c}^{*}}} L_{1}\left(\phi_{c}\right) .
$$

Completing the definition, $A$ (or $\left(A, N^{A}\right)$ ) will be called an abstract Morse Transue (AMT) space if

(i) $A \subset \Omega^{A}$.

(ii) For every $\phi \in A_{\bar{c}}^{*}, \phi=\phi_{c}^{*}=\phi_{c *}$ on $A$.

(iii) $A^{*}=A_{\bar{c}}^{*}$.

These conditions correspond respectively to the Morse-Transue conditions that: every $x \in A$ is integrable with respect to every measure in the measure dual, $\phi(x)$ can be recovered by integrating with respect to the measure corresponding to $\phi$, and the measure dual is isomorphic to all of $A^{*}$. Clearly (ii) implies (i).

Theorem 3.1. $\left(A, N^{A}\right)$ is an AMT space (with respect to $C$ and $R$ ) if and only if for every $\phi \in A^{*}, \phi_{c}{ }^{*}=\phi_{c *}=\phi$ on $A$.

All that needs to be verified is that these conditions imply (iii). If $\phi \in A^{*}$ and $u_{n} \downarrow 0(R)$ with $u_{n} \in A, n=1,2, \ldots, \phi_{c *}\left(u_{n}\right) \rightarrow 0$ by $\mathrm{N} 9$ so that $\phi\left(u_{n}\right) \rightarrow 0$ and $\phi \in A^{*}$.

Theorem 3.2. If $A$ is an AMT space, $C$ is $N^{A}$-dense in $A$.

Proof. Assume the theorem to be false. Then the Hahn-Banach theorem asserts the existence of a point $x_{0} \in A-C$ and $\phi^{\prime} \in A^{*}$ with $\phi^{\prime}(x)=0$ in $C$, $\phi^{\prime}\left(x_{0}\right) \neq 0$. Then $\phi_{c}^{\prime}=0$ on $C,{\phi^{\prime}}_{c}^{*}=\phi_{c *}^{\prime}=0$ on $A$, contradicting (ii).

THEOREM 3.3. If $\left(A, N^{A}\right)$ is an AMT space, $u_{n} \downarrow 0(R)$ implies that $N^{A}\left(u_{n}\right) \rightarrow 0$.

Proof. Since $A^{*}=A_{\bar{c}}^{*}, u_{n} \downarrow 0(R)$ implies that $\phi\left(u_{n}\right) \rightarrow 0$ for every $\phi \in A^{\prime}$, i.e. $\left\{u_{n}\right\}$ converges weakly to zero. The argument of (3, VII, Lemma 22.6, p. 671) then applies to complete the proof.

Corollary. If $\left(A, N^{A}\right)$ is an AMT space and $u_{n} \uparrow u(R)$ in $A^{+}$, then $N^{A}\left(u_{n}\right) \uparrow N^{A}(u)$.

Examples. For any Riesz seminorm $N^{c},\left(C, N^{C}\right)$ is an AMT space if and only if $C^{*}=C_{\bar{c}}^{*}$. Since $C^{*} \subset C^{\sim}$, this is always the case if $C^{\sim}=C_{\bar{c}}$.

If $C$ is too small, the spaces in the preceding paragraph may be the only AMT spaces for $C$ and $R$. For example, let $X$ be an arbitrary set of points, $R=\Re^{x}$, where $\Re$ denotes the reals and $R$ has the natural partial order. Let $C$ 
denote the constant functions on $X$. Then $C^{\sim}=C_{\bar{c}} \sim$ is isomorphic to $\Re$. Let $u(x)=1$ in $X$. Then for each $\phi \in C^{\sim}, f \in R$,

$$
\phi^{*}(f)=\left(\sup _{x \in X} f(x)\right) \phi(u)>\left(\inf _{x \in X} f(x)\right) \phi(u)=\phi_{*}(f)
$$

unless $f \in C$. Thus for any $\left(A, N^{A}\right), \Omega^{A}=C$ and (i) implies that no $A$ larger than $C$ can be an AMT space.

For every $\phi \in C_{\bar{c}}^{-},\left(L_{1}(\phi), \rho \varphi\right)$ is an AMT space.

We can assume that $\phi>0$. Since $L_{1}(\phi)$ is $\sigma$-Dedekind complete, $u_{n} \downarrow f$ means the same in $R$ and $L_{1}(\phi)$. Since $\rho \varphi$ and $\phi=\phi_{*}$ coincide on $L_{1}(\phi)^{+}$,

$$
\rho \varphi\left(u_{n}\right) \downarrow 0 \quad \text { if } u_{n} \downarrow 0,
$$

by N9. This implies that every $\phi^{\prime}$ in $L_{1}(\phi)^{*}$ is in $L_{1}\left(\phi^{*}\right)$ so that (iii) holds.

We next note that if $0<\phi^{\prime} \in L_{1}(\phi)^{*}, L_{1}\left(\phi_{c}^{\prime}\right)>L_{1}(\phi)$. Since $\phi_{c}^{\prime} \leqslant\left\|\phi^{\prime}\right\| \phi$ on $C^{+}, \phi_{c}^{\prime}{ }_{c}^{*} \leqslant\left(\left\|\phi^{\prime}\right\| \phi\right)^{*}=\left\|\phi^{\prime}\right\| \phi^{*}$ on $L_{1}(\phi)^{+} \subset R^{+}$by Theorem 2.1 .

If $f \in L_{1}(\phi)$ and $v \in C, 2.3$ implies that

$$
\begin{aligned}
0 \leqslant \phi_{c}^{\prime}(f)-\phi_{c *}^{\prime}(f) & =\phi_{c}^{\prime}{ }^{*}(f-v)+\phi_{c}^{\prime}{ }^{*}(v-f) \\
& \leqslant 2 \phi_{c}^{\prime}{ }^{*}(|f-v|) \leqslant 2\left\|\phi^{\prime}\right\| \phi(|f-v|) .
\end{aligned}
$$

Since $C$ is $\rho \varphi$-dense in $L_{1}(\phi),{\phi^{\prime}}_{c}^{*}(f)={\phi^{\prime}}_{c *}(f)$ and $f \in L_{1}\left(\phi^{\prime}{ }_{c}\right)$.

To prove (ii) we note that for every $u \in C, f \in L_{1}(\varphi)$,

$$
\begin{aligned}
\left|\phi_{c}^{\prime}(f)-\phi^{\prime}(f)\right| & \leqslant\left|\phi_{c}^{\prime}(f)-\phi_{c}^{\prime}(u)\right|+\left|\phi^{\prime}(f)-\phi^{\prime}(u)\right| \\
& \leqslant 2|| \phi^{\prime}|| \phi(|f-u|)
\end{aligned}
$$

and the right side can be made arbitrarily small by choice of $u \in C$.

4. Extensions of AMT spaces and normed AMT spaces. Let $\left(A, N^{A}\right)$ be an arbitrary AMT space. From Banach space theory (iii) implies that for all $f \in A$,

$$
N^{A}(f)=\sup _{\substack{\varphi \in A^{*} \\\|\varphi\| \leqslant 1}}|\phi(f)| .
$$

By (3, VII, Lemma 22.3), $\|\phi\|=\||\phi|\|$ and, since $N^{A}(f)=N^{A}(|f|)$,

$$
N^{A}(f)=\sup _{\substack{\phi \in A^{*} \\ \| \phi|| \leqslant 1}}|\phi|(|f|)=\sup _{\substack{\phi \in A^{*+} \\\|\phi\| \leqslant 1}} \phi(|f|) .
$$

Using this formula, $N^{A}$ can be extended from $A$ to $\Omega^{A}$. Replacing $\phi$ by $\phi^{*}$ in $\varphi(|f|)$ gives an extension to all of $R$ (valued in the non-negative extended reals). We shall use the notation $N^{A}$ also for the above extension. Then, on $R, N^{A}$ satisfies:

4.1. $0 \leqslant N^{A}(f) \leqslant+\infty$,

4.2. $N^{A}(a f)=a N^{A}(f), a \geqslant 0$,

4.3. $N^{A}(f+g) \leqslant N^{A}(f)+N^{A}(g)$,

4.4. $N^{A}(f) \leqslant N^{A}(g)$ if $|f| \leqslant|g|$,

4.5. $N^{A}\left(f_{n}\right) \uparrow_{n=1}^{\infty} N^{A}(f)$ if $0 \leqslant f_{n} \uparrow f$. 
From 4.4 we see that $N^{A}(f)=N^{A}(|f|)$. Property 4.5 is an easy consequence of $\mathrm{N} 8$ which is valid for all $\phi \in A^{*+}$.

Let $F^{A}=\left[f \in R: N^{A}(f)<\infty\right]$. Then $F^{A}$ is an ideal of $R$ and thus is $\sigma$ Dedekind complete. $N^{A}$ is a Riesz seminorm on $F^{A}$. $F^{A}$ is closed for the seminorm topology.

Set $\Phi^{A}=\left[f \in R: N^{A}(f)=0\right]$. Clearly $\Phi^{A} \subset \Omega^{A}, F^{A}$.

Now $f=g$ if $f-g \in \Phi^{A}$ defines equivalence relations on $C, A, \Omega^{A}, F^{A}$, and $R$. Using boldface type for equivalence classes and spaces of equivalence classes,

$$
\begin{aligned}
\mathbf{f} & =f+\Phi^{A} \text { in } R, F^{A}, \text { and } \Omega^{A} ;=f+\Phi^{A} \cap A \text { in } A ; \\
& =f+\Phi^{A} \cap C \text { in } C .
\end{aligned}
$$

Then $N^{A}$ is uniquely defined on $\mathbf{R}$ and is a norm on $\mathbf{C}, \mathbf{A}$, and $\mathbf{F}^{A}$. Partially $\operatorname{order} \mathbf{R}$ by $f \leqslant g$ if, for each $f \in \mathbf{f}, g \in \mathbf{g},(f-g)^{+} \in \Phi^{A}$. Then $\mathbf{R}$ is $\sigma$-Dedekind complete and $\mathbf{G}$ and $\mathbf{A}$ are Riesz subspaces of $\mathbf{R}$ with $\mathbf{C} \subset \mathbf{A} \subset \mathbf{R}$. We shall show that $\left(\mathbf{A}, N^{A}\right)$ is a normed AMT space with respect to $\mathbf{G}$ and $\mathbf{R}$.

Now $A^{*}$ and $\mathbf{A}^{*}$ consist of the same elements. Suppose that $\mathbf{u}_{n} \downarrow \mathbf{0}(R)$. We shall show that for each $\varphi \in A^{*}$ there then exists a sequence $u_{n}^{\prime} \downarrow 0(R)$ with $\left\{u_{n}^{\prime}\right\} \subset L_{1}(\varphi), u_{n}^{\prime} \in u_{n}, n=1,2, \ldots$ Since $A^{*}=A_{c}^{*}$ by assumption, this will imply that (iii) holds for $\mathbf{A}$.

We first note that if $u \in \mathbf{u}$ and $\mathbf{u} \geqslant \mathbf{0}$, then $u^{-} \in \Phi^{A} \cap A$ and $u^{+} \in \mathbf{u}$. Let $u_{1}>0$ be an arbitrary element of $\mathbf{u}_{1}, u_{2}$ any element of $\mathbf{u}_{2}$. Since $\mathbf{u}_{1} \geqslant \mathbf{u}_{2}$, $\left(u_{2}-u_{1}\right)^{+} \in \Phi^{A} \cap A$. Set $u_{2}^{\prime}=\left[u_{2}-\left(u_{2}-u_{1}\right)^{+}\right]^{+}$. Then $u_{2}^{\prime} \in A$ and $u_{1}^{\prime}=u_{1} \geqslant u_{2}$. The remaining elements ${u^{\prime}}_{n}$ are determined similarly by induction. Let $v=\wedge u_{n}^{\prime}$, which exists in $R$ since $R$ is $\sigma$-Dedekind complete. Since $\mathbf{0}=\wedge \mathbf{u}_{n}, \mathbf{v} \leqslant \mathbf{0}$ and $v^{+} \in \Phi^{A}$. Let $u_{n}^{\prime \prime}=u_{n}^{\prime}-v^{+}$. Then $u_{n}^{\prime \prime}{ }_{n} \downarrow 0$ in $R$ and in $L_{1}(\phi)$ for every $\phi \in A^{*}$. It follows that $\lim \phi\left(\mathbf{u}_{n}\right)=\lim \phi\left(u_{n}^{\prime \prime}\right)=0$ and $\phi \in \mathbf{A}_{\bar{c}}^{*}$.

We next show that (ii) holds for every $\phi \in \mathbf{A}^{*}=\mathbf{A}_{c}^{*}$. Let $u \in \mathbf{u} \in A$, $\phi \in \mathbf{A}^{*+}=A^{*+}$. Then $\phi_{c}^{*}(\mathbf{u})=\phi_{c}^{*}(u)=\phi_{c *}(u)=\phi_{c *}(\mathbf{u})$.

In the analogous MT case $F^{A}$ is always $N^{A}$-complete. Let $C=A=R$ denote the bounded Lebesque integrable functions on $(0,1)$ seminormed by

$$
N_{1}(f)=\int_{0}^{1}|f| d x
$$

and with identifications. Then $A$ is an AMT space and $F^{A}=R$ is not complete. That this example is typical is illustrated by the following theorem.

THEOREM 4.1. The normed space $\mathbf{F}^{A}$ is $N^{A}$-complete if and only if $\mathbf{u}_{n} \geqslant 0$, $n=1,2, \ldots$,

$$
\sum_{1}^{\infty} N^{A}\left(\mathbf{u}_{n}\right)<\infty \quad \text { implies that } \quad \sum_{1}^{\infty} \mathbf{u}_{n} \in \mathbf{R} \text {. }
$$


Proof. Completeness in norm implies this condition with $\sum_{1}^{\infty} \mathbf{u}_{n}$ in $\mathbf{F}^{A}$ by (3, VIII, Theorem 26.3). Conversely, if the condition is satisfied, $\sum_{1}^{m} \mathbf{u}_{n} \uparrow \sum_{1}^{\infty} \mathbf{u}_{n}$ so that, by 4.5 and 4.3 ,

$$
N^{A}\left(\sum_{1}^{\infty} \mathbf{u}_{n}\right)=\lim _{m \rightarrow \infty} N^{A}\left(\sum_{1}^{m} \mathbf{u}_{n}\right) \leqslant \sum_{\mathbf{1}}^{\infty} N^{A}\left(\mathbf{u}_{n}\right)<\infty,
$$

and $\sum_{1}^{\infty} \mathbf{u}_{n} \in \mathbf{F}^{A}$. The theorem is then completed by applying Theorem 26.3 quoted above.

Theorem 4.2. If $f \in R$ and $N^{A}(f)=+\infty, f \notin \Omega^{A}$. Thus $\Omega^{A} \subset F^{A}$. (Compare (5, Theorem 5.1, p. 351).)

Proof. By definition

$$
N^{A}(f)=\sup _{\substack{\varphi \in A^{*}+\\|\varphi| \mid \leqslant 1}} \phi^{*}(|f|)
$$

The hypothesis implies the existence in $A^{*+}$ of a sequence $\left\{\phi_{n}\right\}$ with

Set

$$
\phi_{n}{ }^{*}(|f|)>2^{n}, \quad\left\|\phi_{n}\right\| \leqslant 1, \quad n=1,2, \ldots
$$

$$
\phi^{\prime}=\sum_{n=1}^{\infty} \phi_{n} / 2^{n}
$$

It is easily verified that $\phi^{\prime} \in A^{*+}$ with $\left\|\phi^{\prime}\right\| \leqslant 1$.

By Theorem 2.1 , for $N=1,2, \ldots$,

Thus

$$
\phi^{\prime} \geqslant\left(\sum_{1}^{N} \phi_{n} / 2^{n}\right) *=\sum_{1}^{N} \phi_{n}^{*} / 2^{n} \quad \text { on } R^{+} .
$$

$$
\phi^{*}(|f|) \geqslant \sum_{1}^{N} \phi_{n}^{*}(|f|) / 2^{n}>N
$$

for every $N$. Thus $f \notin L_{1}\left(\phi^{\prime}\right)$ and therefore not in $\Omega^{A}$.

5. Compatible AMT spaces. Two AMT spaces $\left(A, N^{A}\right)$ and $\left(B, N^{B}\right)$ (with respect to the same $C$ and $R$ ) will be called compatible if $A^{*}=B^{*}$ and $N^{A}=N^{B}$ on $C$.

When $A$ is not $\sigma$-Dedekind complete we let $A^{\prime+}=\left[f \in R: f=\wedge u_{n}\right.$ (in $R$ ) for some sequence $\left.\left\{u_{n}\right\} \subset A^{+}\right]$and set $A^{\prime}=A^{\prime+}-A^{\prime+}$. Then $A^{\prime}$ is the smallest $\sigma$-Dedekind complete Riesz subspace of $R$ containing $A$. If $f \in A^{\prime+}$, there exist sequences $\left\{u_{n}\right\},\left\{v_{n}\right\}$ in $A^{+}$with $u_{n} \downarrow f, v_{n} \uparrow f$ (in $R$ ).

Lemma 5.1. $A^{\prime} \subset \Omega^{A}$.

Proof. Let $\phi \in A^{*+}, f \in A^{\prime+}, u_{n} \downarrow f, v_{n} \uparrow f$ (in $R$ ) with each $u_{n}, v_{n}$ in $A^{+}$. Now

$$
\phi^{*}(f)-\phi_{*}(f)=\phi^{*}(f)-\phi\left(v_{n}\right)+\phi\left(v_{n}-u_{n}\right)-\phi_{*}(f)-\phi\left(u_{n}\right) .
$$

Since $u_{n}-v_{n} \downarrow 0(R), \phi\left(u_{n}-v_{n}\right) \rightarrow 0$. Furthermore $\phi^{*}(f)-\phi\left(v_{n}\right) \rightarrow 0$ by N8 and $\phi_{*}(f)-\phi\left(u_{n}\right) \rightarrow 0$ by N9. Thus $\phi^{*}(f)=\phi_{*}(f)$ and both are finite since $f$ is bounded above in $A$. Thus $f \in L_{1}(\phi)$ for each positive $\phi$ and therefore is in $\Omega^{A}$. 
Theorem 5.1. $\left(A, N^{A}\right)$ and $\left(A, N^{\prime} N^{\prime}\right)$ are compatible AMT spaces.

Proof. Here $N^{A^{\prime}}$ denotes the extension of $N^{A}$ from $A$ to $A^{\prime}$. It is thus sufficient to show that $A^{*}=A^{\prime *}$. If $\phi \in A^{*}, \phi^{*}=\phi_{*}$ is linear on $A^{\prime}$ since $A^{\prime} \subset L_{1}(\phi)$. Furthermore, where $0 \leqslant u_{n} \uparrow f, u_{n} \in A, f \in A^{\prime}$,

$$
\begin{aligned}
\left|\phi^{*}(f)\right| \leqslant|\phi|^{*}(f) & =\lim _{n \rightarrow \infty}|\phi|\left(u_{n}\right) \leqslant\|\phi\| \lim N^{A}\left(u_{n}\right) \\
& =\|\phi\| N^{A}(f),
\end{aligned}
$$

using 4.5. Since $\geqslant$ is obvious, $\left\|\phi^{*}\right\|_{A^{\prime} *}=\|\phi\|_{A *}$. From N9 it follows that $\phi^{*} \in A_{c}{ }^{*}$.

Conversely, each $\phi$ in $A^{*+}$ determines a $\phi^{\prime} \in A^{*}=A_{\bar{c}}^{*}$ by restriction to $A$. From linearity it follows that $\phi$ is monotone on $A^{\prime+}$. Thus if $f>0, u_{n} \downarrow f$, $v_{n} \uparrow f,\left\{u_{n}\right\},\left\{v_{n}\right\} \subset A, \phi\left(f-v_{n}\right), \phi\left(u_{n}-f\right) \leqslant \phi^{\prime}\left(u_{n}-v_{n}\right)$. Since $u_{n}-v_{n} \downarrow 0(R)$, $\phi^{\prime}\left(u_{n}-v_{n}\right) \rightarrow 0$ and $\phi(f)=\lim \phi\left(u_{n}\right)=\lim \phi\left(v_{n}\right)$. It follows that $\phi=\phi^{\prime *}$ on $A^{\prime}$ so that the correspondence between $A^{*}$ and $A^{\prime *}$ is 1-1 and norm-preserving. This implies that $\left(A^{\prime}, N^{A^{\prime}}\right)$ is an AMT space and that this space is compatible with $\left(A, N^{A}\right)$.

Theorem 5.2. (i) $\left(\mathbf{A}^{\prime}, N^{A}\right)$ is super Dedekind complete and if $f_{a}, a \in \mathfrak{A}$, is a system of elements of $\mathbf{A}^{\prime+}$ filtering down to zero, $\mathbf{f}_{a} \downarrow_{a \in \mathfrak{a}} \mathbf{0}$, then $N^{A}\left(\mathbf{f}_{a}\right) \downarrow 0$. (ii) Every order-bounded increasing sequence in $A^{\prime}$ has a norm limit.

Proof. By Theorem 3.3 if $\mathbf{f}_{n} \downarrow \mathbf{0}, \mathbf{f}_{n} \in \mathbf{A}^{\prime}, N^{A}\left(\mathbf{f}_{n}\right) \downarrow 0$. Since $\mathbf{A}^{\prime}$ is $\sigma$-Dedekind complete, the conditions of $(3, \mathrm{X}$, Theorem 33.4, p. 503$)$ are satisfied.

If $\left(A, N^{A}\right)$ is an AMT space for $C$ and $R,\left(C, N^{A}\right)$ is compatible with $\left(A, N^{A}\right)$. This follows from the definition. Obviously $\left(C, N^{A}\right)$ is the smallest AMT space compatible with $\left(A, N^{A}\right)$.

Let $\bar{A}$ denote the closure of $A$ in $F^{A}$ for the $N^{A}$ seminorm topology. (Compare $(4, \S 13)$.)

Theorem 5.3. $\left(\bar{A}, N^{A}\right)$ is an AMT space contained in $\Omega^{A}$ and compatible with $\left(A, N^{A}\right)$ and is maximal in the sense that every AMT space compatible with $\left(A, N^{A}\right)$ is contained in $\left(\bar{A}, N^{A}\right)$.

Proof. We first note that $\bar{A}$ is a Riesz subspace of $R$. Let $u \in \bar{A}$. Since $C$ is dense in $A$, there exist $u_{n} \in C$ with

$$
\lim _{n \rightarrow \infty} N^{A}\left(u-u_{n}\right)=0 .
$$

It follows easily that $\bar{A}$ is linear. Now $|u| \in R$ and each $\left|u_{n}\right| \in C$. Thus, since

$$
\begin{gathered}
|| u|-| u_{n}||=\left(|u|-\left|u_{n}\right|\right)^{+}+\left(|u|-\left|u_{n}\right|\right)^{-} \\
\leqslant 2\left(\left|u-u_{n}\right|\right)^{+}=2\left|u-u_{n}\right|, \\
\lim _{n \rightarrow \infty} N^{A}\left(|u|-\left|u_{n}\right|\right)=0,
\end{gathered}
$$

and $|u| \in \bar{A}$. This implies that $u^{+}$and $u^{-} \in \bar{A}$ and $\bar{A}$ is closed under the lattice operations.

On $\bar{A}$

$$
N^{A}(u)=\sup _{\substack{\phi \in A^{*} \\|\| \phi| \leqslant 1}} \phi^{*}(|u|)
$$


With $u, u_{n}$ as in the preceding paragraph, and each $\phi \in A^{*+}$, using 2.3,

$$
\phi^{*}(u)-\phi_{*}(u)=\phi^{*}\left(u-u_{n}\right)+\phi^{*}\left(u_{n}-u\right) \leqslant 2\|\phi\| N^{A}\left(u-u_{n}\right),
$$

$n=1,2, \ldots$ It follows that $u \in \Omega^{A}, \bar{A} \subset \Omega^{A}$. Thus if $\phi \in A^{*}, \phi=\phi^{*}$ is linear on $A$. By continuity, $\phi$ is bounded on $\bar{A}$ with the same norm as on $A$. Using N9, each $\phi \in A^{*}$ extends to $\phi \in \bar{A}_{\bar{c}}^{*}$.

Let $\phi \in \bar{A}^{*}, \phi^{\prime}=\phi \mid A$. Then $\phi^{\prime}$ extends to $\bar{\phi}^{\prime}$ on $\bar{A}$. Let $u \in \bar{A}, N^{A}\left(u-u_{n}\right)$ $\rightarrow 0, u_{n} \in C$. Then

$$
\left|\phi(u)-\bar{\phi}^{\prime}(u)\right|=\left|\phi\left(u-u_{n}\right)+\bar{\phi}^{\prime}\left(u_{n}-u\right)\right| \leqslant\left(\|\phi\|-\| \phi^{\prime}||\right) N^{A}\left(u-u_{n}\right),
$$

$n=1,2, \ldots$, and $\phi=\bar{\phi}^{\prime}$.

Corollary 1. $\bar{A}$ is complete if $F^{A}$ is complete.

Corollary 2. If $A=L_{1}(\phi), A=\bar{A}$.

In this case $A=\Omega^{A}$ and is therefore maximal.

Corollary $3 . \overline{\mathbf{A}} \supset \mathbf{A}^{\prime}$.

THEOREM 5.4. Every $\mathbf{f} \in \mathbf{\Omega}^{A}$ that is majorized above and below by elements of $\mathbf{A}^{\prime}$ (equivalently of $\mathbf{G}$ ) is in $\mathbf{A}^{\prime}$, i.e. $\mathbf{A}^{\prime}$ is an ideal of $\mathbf{\Omega}^{A}$.

Proof. Let $\mathbf{f} \in \mathbf{\Omega}^{A+}$ and suppose that there exists $\mathbf{v} \in \mathbf{C}$ with $\mathbf{f} \leqslant \mathbf{v}$. Choose $0 \leqslant v \in \mathbf{v} \cap \mathbf{C}, f \in \mathbf{f} \cap \mathbf{A}^{\prime}$ with $0 \leqslant f \leqslant v$, and let $\boldsymbol{\phi} \in A^{*+}$. Then $\boldsymbol{\phi}^{*}(f)<\infty$ and there exist sequences $u_{n i} \uparrow_{i=1}^{\infty}$ with $u_{n i} \wedge f \uparrow_{i} f$ and

$$
\lim _{i \rightarrow \infty} \phi\left(u_{n i}\right) \geqslant \phi^{*}(f) \geqslant \lim _{i \rightarrow \infty} \phi\left(u_{n i}\right)-1 / n, \quad n=1, \ldots
$$

The sequences can be assumed to be bounded above by $v$ and thus $f_{n}=\vee_{i} u_{n} i$ exists in $A^{\prime}$ since $A^{\prime}$ is $\sigma$-Dedekind complete. Using Lemma 2.1 and induction, it can be assumed that $f \leqslant f_{n} \downarrow_{n}$. Thus

$$
\phi^{*}(f) \leqslant \phi\left(f_{n}\right) \leqslant \phi^{*}(f)-1 / n, \quad n=1,2, \ldots,
$$

and $\lim _{n \rightarrow \infty} \phi\left(f_{n}\right)=\phi^{*}(f)$.

Let $f_{\varphi}=\wedge f_{n}$. Then $\phi^{*}\left(\mathbf{f}_{\varphi}\right)=\phi^{*}(\mathbf{f})$. Since $\mathbf{A}^{\prime}$ is Dedekind complete by Theorem 5.2, $\mathbf{f}^{\prime}=\wedge_{\varphi \in A^{*+}} \mathbf{f}_{\varphi}$ exists in $\mathbf{A}^{\prime}$ with $\mathbf{f}^{\prime} \geqslant \mathbf{f}$. Since $\boldsymbol{\Omega}^{A} \subset \mathbf{L}_{\mathbf{1}}(\phi)$, each $\phi$ is linear on $\mathbf{\Omega}^{A}$. Thus

$$
\phi^{*}\left(\mathbf{f}^{\prime}-\mathbf{f}\right)=\phi^{*}\left(\mathbf{f}^{\prime}\right)-\phi^{*}(\mathbf{f})=0,
$$

for each $\phi \in A^{*+}, N^{A}\left(\mathbf{f}^{\prime}-\mathbf{f}\right)=0$ and $\mathbf{f}^{\prime}=\mathbf{f} \in \mathbf{A}^{\prime}$.

THEOREM 5.5. $\overline{\mathbf{A}}$ is an ideal of $\mathbf{\Omega}^{A}$. If every increasing sequence of elements of $\mathbf{A}^{\prime+}$ that is bounded in norm is a Cauchy sequence, then $\overline{\mathbf{A}}=\mathbf{\Omega}^{A}$. Theorem 5.2 extends to $\bar{A}$.

Proof. By Theorem 3.3, if $\mathbf{u}_{n} \downarrow \mathbf{0}, \mathbf{u}_{n} \in \overline{\mathbf{A}}$, then $N^{A}\left(\mathbf{u}_{n}\right) \downarrow 0$ as $n \rightarrow \infty$. If $\mathbf{f} \in \overline{\mathbf{A}}^{+}, N^{A}(\mathbf{f})<\infty$ and there exists $\mathbf{u}_{n} \wedge \mathbf{f} \uparrow \mathbf{f}$, with $\mathbf{u}_{n} \in \mathbf{C}$. By the preceding theorem $\mathbf{u}_{n} \wedge \mathbf{f} \in \mathbf{A}^{\prime} \subset \overline{\mathbf{A}}$. Then $\mathbf{f}-\mathbf{u}_{n} \wedge \mathbf{f} \downarrow \mathbf{0}$ so that $N^{A}\left(\mathbf{f}-\mathbf{u}_{n} \wedge \mathbf{f}\right)$ $\rightarrow 0$ as $n \rightarrow \infty$. If $0<\mathbf{g} \leqslant \mathbf{f}$,

$$
\mathbf{g}-\mathbf{u}_{n} \wedge \mathbf{g}=\left(\mathbf{g}-\mathbf{u}_{n}\right)^{+} \leqslant\left(\mathbf{f}-\mathbf{u}_{n}\right)^{+}=\mathbf{f}-\mathbf{u}_{n} \wedge \mathbf{f}
$$


and $N^{A}\left(g-\mathbf{u}_{n} \wedge \mathbf{g}\right) \rightarrow 0$ as $n \rightarrow \infty$. Thus $\mathbf{g} \in \overline{\mathbf{A}}^{\prime}=\overline{\mathbf{A}}$. It follows that $\overline{\mathbf{A}}$ is an ideal of $\boldsymbol{\Omega}^{A}$.

If $\mathbf{f} \in \mathbf{\Omega}^{A}, N^{A}(\mathbf{f})<\infty$ by Theorem 4.2. There then exists a sequence $\left\{\mathbf{u}_{n} \wedge \mathbf{f}\right\}$ in $\mathbf{A}^{\prime}$ with $\mathbf{u}_{n} \wedge \mathbf{f} \uparrow \mathbf{f}$. By 4.5

$$
N^{A}\left(\mathbf{u}_{n} \wedge \mathbf{f}\right) \uparrow N^{A}(\mathbf{f}) .
$$

Since $\mathbf{u}_{n} \wedge \mathbf{f}$ is a Cauchy sequence by hypothesis, $N^{A}\left(\mathbf{f}-\mathbf{u}_{n} \wedge \mathbf{f}\right) \rightarrow 0$ as $n \rightarrow \infty$ and $\mathbf{f} \in \overline{\mathbf{A}}^{\prime}=\overline{\mathbf{A}}$. (Compare (5, Theorem 7.2, p. 359).)

Theorem 5.6. $\left(\boldsymbol{\Omega}^{A}, N^{A}\right)$ is an AMT space if and only if $\boldsymbol{\Omega}^{A}=\overline{\mathbf{A}}$.

Proof. If $\boldsymbol{\Omega}^{A}=\overline{\mathbf{A}}$, Theorem 5.3 applies. Now $\boldsymbol{\Omega}^{A}$ is a Riesz subspace of $\mathbf{R}$ normed by $N^{A}$. Since $\overline{\mathbf{A}} \subset \boldsymbol{\Omega}^{A}$ and compatibility is an equivalence relation, $\left(\mathbf{\Omega}^{A}, N^{A}\right)$ cannot be compatible with $\left(\mathbf{A}, N^{A}\right)$ if $\boldsymbol{\Omega}^{A} \neq \overline{\mathbf{A}}$. For each $\phi$ in $A^{*}$ the Nakano extension gives an extension $\phi^{*}=\phi_{*}$ on $\boldsymbol{\Omega}^{A}$ that is $N^{A}$-bounded. If $\phi \in \boldsymbol{\Omega}^{A *+}, \phi$ determines $\phi^{\prime} \in A^{*}$ by restriction to $\mathbf{A}$ and $\phi^{\prime}$ extends to $\boldsymbol{\Omega}^{A}$. Since $\boldsymbol{\Omega}^{A}$ is linear, non-compatibility implies the existence of some $\bar{\phi}=\phi-\phi^{\prime}$ in $\mathbf{\Omega}^{A *}$ with $\bar{\phi}=0$ on $\mathbf{A}^{\prime}$. We can assume that $\bar{\phi}>0$ and that there exists $\mathbf{x}$ with $\bar{\phi}(\mathbf{x})>0$. As in the previous theorem, there exists $\mathbf{u}_{n} \uparrow \mathbf{x}$ with each $\mathbf{u}_{n} \in \mathbf{A}^{\prime}$. Thus $\mathbf{x}-\mathbf{u}_{n} \downarrow \mathbf{0}$ but $\bar{\phi}\left(\mathbf{x}-\mathbf{u}_{n}\right)=\bar{\phi}(\mathbf{x})$ for all $n$. Thus $\bar{\phi} \in \Omega^{A *}$ and not in $\Omega_{\bar{c}}^{A *}$, condition (iii) is violated, and $\boldsymbol{\Omega}^{A}$ is not an AMT space.

The following example illustrates the preceding result. Compare (3, XI, p. 509). Let $R$ denote the space of sequences $x=\left(x_{1}, x_{2}, \ldots\right) ; C=c_{0}$, the space of sequences convergent to zero; $A=C, N^{A}$ the uniform norm on $A$. Then $A=A^{\prime}=\bar{A}$ is norm complete so that $C^{\sim}=C^{*}(3$, VIII, Theorem 26.4). It is easy to show that $C^{*}$ is equivalent to $l_{1}$ by the correspondence $y(x)=\sum x_{i} y_{i}$. If $u_{n} \downarrow 0, N^{A}\left(u_{n}\right) \downarrow 0$ as $n \rightarrow \infty$. This implies that $C^{\sim}=C_{\bar{c}}^{\sim}$ and, noting that $C$ is $\sigma$-Dedekind complete (actually super Dedekind complete),

$$
C^{\sim}=C_{c}^{\sim}=C_{\bar{c}}^{\sim}=C^{*}=l_{1}
$$

Thus $\left(C, N^{A}\right)$ is a normed AMT space.

Let $0<y \in C^{*} ; 0<x \in R, x^{n}=\left(x_{1}^{n}, x_{2}^{n}, \ldots\right), x_{i}^{n}=x_{i}, i=1,2, \ldots, n$; $=0, i>n$. Then $y^{*}(x)=y_{*}(x) \leqslant \infty$ on $R^{+}$by 2.1. The extended $N^{A}$ is given by

$$
N^{A}(x)=\sup _{i}\left|x_{i}\right|,
$$

and $\Omega^{A}=F^{A}=l \infty$. The Hahn-Banach theorem implies the existence of Banach generalized limits $\phi(x)=(\mathrm{H}-\mathrm{B}) \lim x_{n}$ with

$$
\varlimsup_{\lim } x_{n} \geqslant \phi(x) \geqslant \underline{\lim }_{n \rightarrow \infty} x_{n}
$$

$0 \neq \phi \in(l \infty)^{*}$. For every $x \in C=\bar{A}, \phi(x)=0$.

6. Real MT spaces as AMT spaces. In this section we let $E$ be an arbitrary locally compact space, $C$ the functions continuous on $E$ with compact supports, 
and $R=\Re^{E}$. Then $C^{\sim}$ corresponds to the space of all Radon measures on $E$ and $C^{\sim}=C_{\bar{c}}^{\sim}$.

For $\phi \in C^{\sim+}$ we consider $L_{1}(\phi)=L_{1}\left(\phi^{*}\right)$ under the norm $N^{A}=\rho \varphi$ extended by $N^{A}(u)=\varphi^{*}(|u|)$ to $R$. It is well known that $L_{1}\left(\phi^{*}\right)$ is complete under $\rho \varphi$, but we include a proof adapted to the present context. We shall show that the hypotheses of Theorem 4.1 are satisfied so that $\mathbf{F}^{A}$ is complete and thus $L_{1}(\phi)$ is complete since it is norm closed in $\mathbf{F}^{A}$.

Let $\mathbf{u}_{n}>\mathbf{0}, n=1,2, \ldots, \sum_{1}^{\infty} \phi^{*}\left(\mathbf{u}_{n}\right)<\infty$. There exist $u_{n} \in \mathbf{u}_{n}, u_{n}>0$, $u_{n} \uparrow_{n}$ and thus there exists $u(t)$ valued in $\bar{\Re}^{+}$with $u_{n}(t) \uparrow u(t)$ for all $t \in E$. Let $E_{0}=[t: u(t)<\infty], u^{\prime}(t)=u(t) \chi_{E_{0}}$. Then $u^{\prime} \in R^{+}, \sum_{1}^{n} u_{i} \chi_{E_{0}} \uparrow u^{\prime}$ and, from 4.5 and $4.3, \phi^{*}\left(u^{\prime}\right) \leqslant \sum_{1}^{\infty} \phi^{*}\left(u_{n}\right)<\infty$ and $\mathbf{u}^{\prime} \in \mathbf{F}^{A}$. To show that $\mathbf{u}^{\prime}=$ $\sum_{1}^{\infty} \mathbf{u}_{n}$ we show that for each $n, u_{n}-u_{n} \chi_{E_{0}} \in \Phi^{A}$. Suppose that there exists $n_{0}$ with

$$
u^{\prime}=u_{n_{0}} \chi_{E_{0}}, \quad \phi^{*}\left(u^{\prime}\right)=a>0 .
$$

Since $\left(\sum_{1}^{n} u_{i}\right) \wedge\left(K u^{\prime}\right) \uparrow\left(K u^{\prime}\right)$ for every $K, 0<K<\infty$, by 4.5

$$
\lim _{n \rightarrow \infty} \phi^{*}\left(\sum_{1}^{n} u_{i}\right) \geqslant \lim _{n \rightarrow \infty} \phi^{*}\left[\left(\sum_{1}^{n} u_{i}\right) \wedge\left(K u^{\prime}\right)\right]=\phi^{*}\left(K u^{\prime}\right)=K a .
$$

By hypothesis,

$$
\phi^{*}\left(\sum_{1}^{n} u_{i}\right) \leqslant \sum_{1}^{\infty} \phi^{*}\left(u_{i}\right)<\infty \quad \text { for every } n .
$$

Thus we have a contradiction for $n, K$ sufficiently large.

It follows that $\mathbf{L}_{1}(\phi)$ is a completion of $\mathbf{C}$ for $\rho \varphi$. Since this is also the case for the space of equivalence classes of functions integrable with respect to the Radon measure $\phi$ for the Bourbaki theory and the two theories coincide on $C$, both theories give the same spaces of integrable functions. It follows that if $\mathbf{A}$ is a Riesz substance of $\mathbf{R}$ and $\mathbf{C} \subset \mathbf{A} \subset \mathbf{R}$ and $N^{A}$ is a Riesz seminorm on $\mathbf{A}$, then the MT and AMT spaces $\boldsymbol{\Omega}^{A}$ coincide and the MT and AMT extensions of $N^{A}$ coincide on $\boldsymbol{\Omega}^{A}$ and $\left(\mathbf{A}, N^{A}\right)$ is an AMT space if and only if it is an MT space.

We next compare the extensions of $N^{A}$ to $R^{+}$. In the Bourbaki theory (1), $\phi \in C^{\sim}$ is first extended to lower semi-continuous functions valued in $\bar{\Re}^{+}$and then to arbitrary functions valued in $\bar{\Re}^{+}$. We have defined $\phi^{*}$ and $\phi_{*}$ above only for functions valued in $\Re$. The two theories are comparable for equivalence classes of functions if we consider, in the Bourbaki case, only those equivalence classes containing finite functions. We shall use the notation $\int{ }^{*}|\mathbf{f}| d \phi$ for the Bourbaki extensions of $\phi, \mathbf{F}^{A}(B)$ for the $\mathbf{f} \in \mathbf{R}$ with $\int *|\mathbf{f}| d \boldsymbol{\phi}<\infty$. Note that if $\mathbf{f} \in \mathbf{F}^{A}(B), \mathbf{f}$ contains finite elements. We shall show that $\mathbf{F}^{A}=\mathbf{F}^{A}(B)$.

We note first that if $\phi^{*}(f)<\infty, \epsilon>0$, there exist $u_{n} \in C$ with $u_{n} \uparrow, u_{n} \wedge f \uparrow f$ and $\phi^{*}(f)>\lim _{n \rightarrow \infty} \phi\left(u_{n}\right)-\epsilon$.

Now $u=\vee_{n} u_{n}$ exists in $\bar{\Re}^{E}$ and is 1.s.c. with

$$
\begin{gathered}
\int^{*} u d \phi=\lim \int u_{n} d \phi=\lim \phi\left(u_{n}\right)<\phi^{*}(f)+\epsilon, \\
\int^{*} f d \phi=\inf _{\substack{u \geqslant s \\
u \text { l.s.c. }}} \int^{*} u d \phi<\phi^{*}(f)+\epsilon .
\end{gathered}
$$


Since $\epsilon>0$ is arbitrary, $\int * \mathbf{f} d \phi \leqslant \phi^{*}(\mathbf{f})$ and $\mathbf{f} \in \mathbf{F}^{A}$.

By (1, Prop. 5, p. 150) if $f \geqslant 0$ is 1.s.c. and $\int{ }^{*} f d \phi<\infty$, then $\mathbf{f} \in \mathbf{L}_{\mathbf{1}}(\phi)$. This implies that $\phi^{*}(\mathbf{f})=\phi_{*}(\mathbf{f})=\int * \mathbf{f} d \phi$,

$$
\int^{*} \mathbf{f} d \phi=\inf _{\substack{g \geqslant f \\ g \text { l.s.c. }}} \int^{*} \mathbf{g} d \phi=\inf _{\substack{g \geqslant f \\ g \text { l.s.c. }}} \phi^{*}(\mathbf{g}) \geqslant \phi^{*}(\mathbf{f}) .
$$

Thus $\mathbf{F}^{A}=\mathbf{F}^{A}(B)$ and $\int *$ and $\phi^{*}$ coincide on $\mathbf{F}^{A}=\mathbf{F}^{A}(B)$.

We have seen that the theory of AMT spaces (for the $C$ and $R$ in this section) and the theory of MT spaces are equivalent. Thus Theorem 5.6 answers affirmatively a question on p. 116 of (6).

7. $\mathbf{L}^{\lambda}$-spaces as AMT spaces. Let $(X, S, \mu)$ denote an arbitrary $\sigma$-finite measure space, $R$ the measurable functions in $\Re^{x}$ with the natural order, $C$ the Riesz subspace of $R$ of simple functions (i.e. of functions each assuming only a finite number of real values different from zero with each value assumed in a set in $S$ of finite measure). Let $A$ be a Riesz subspace of $R$ containing $C$.

We want to determine when $\mathbf{A}$ is both an AMT space and an $\mathbf{L}^{\boldsymbol{\lambda}}$-space (2). We assume that $N^{A}$ is a non-trivial Riesz seminorm on $\mathbf{A}$. If $N^{A}$ is to extend to a length function, it must satisfy (2, p. 576),

$$
(\mathrm{L} 1)^{\prime} \quad N^{A}\left(\chi_{e}\right)=0 \text { if } \mu(e)=0, e \in S .
$$

By Theorem 3.3 we also need

$$
\text { 6.1. } N^{A}\left(u_{n}\right) \downarrow 0 \text { if } u_{n} \downarrow 0(R), u_{n} \in A, n=1,2, \ldots
$$

We consider $\left(A, N^{A}\right)$ where $N^{A}$ is a Riesz seminorm satisfying (L1) ${ }^{\prime}$ and 6.1 .

Let $\phi \in A^{*+}$. Then $\phi_{c} \in C_{\bar{c}}$. If $f \in R^{+}$, there exists a sequence $\left\{u_{n}\right\} \subset C^{+}$ with $u_{n} \uparrow f$. This implies by 2.1 that $\phi^{*}=\phi_{*}$ on $R^{+}$. If $f \in A$,

$$
\left|\phi^{*}(f)-\phi(f)\right| \leqslant 2 \| \phi|| N^{A}\left(f-u_{n}\right), \quad n=1,2, \ldots,
$$

so that $\phi=\phi^{*}=\phi_{*}$ on $A$ and $\left(A, N^{A}\right)$ is an AMT space by Theorem 3.1.

On $R^{+}$the extended $N^{A}$ satisfies $4.1-4.5$ of $\$ 4$ and $(\mathrm{L} 1)^{\prime}$ and these conditions imply that (L1) in (2) is also satisfied. $N^{A}$ can be extended to all measurable functions valued in $\bar{\Re}^{x}$ by (L5) and is then a length function (2). The space $L^{\lambda}, \lambda=N^{A}$, of equivalence classes of functions modulo $\lambda$-negligible functions is an $L^{\lambda}$-space and is therefore complete (2, Theorem 3.1, p. 579). In the present case

$$
\mathbf{L}^{\lambda}=\mathbf{\Omega}^{A}=\mathbf{F}^{A} \supset \overline{\mathbf{A}} .
$$

From Theorem 5.6, $L^{\lambda}$ is an AMT space if and only if $\boldsymbol{\Omega}^{A}=\overline{\mathbf{A}}$.

Let $\phi \in A^{*+}$. Then $\phi$ defines a set function on $S$ by $\phi(e)=\phi\left(\chi_{e}\right)$. From 6.1 it follows that $\phi$ is countably additive and from $(\mathrm{L} 1)^{\prime}$ that it is absolutely continuous with respect to $\mu$. The Radon-Nikodym theorem then asserts the existence of a positive measurable function $g$ with

$$
\phi(f)=\int f g d \mu \leqslant \infty,
$$


for all $f \in R^{+}$. Both sides are finite on $\mathbf{L}^{\lambda}$ and, where $\lambda^{*}$ is the length function conjugate to $\lambda$, it follows easily that $\|\boldsymbol{\phi}\|=\lambda^{*}(\mathbf{g})$ and $\mathbf{g} \in \mathbf{L}^{\lambda^{*}}$. This implies that $\left(L^{\lambda}\right)^{*}=L^{\lambda^{*}}$.

\section{REFERENCES}

1. N. Bourbaki, Éléments de mathématique, fasc. XIII: Integration, chap. I-IV (Paris, 1952).

2. H. W. Ellis and Israel Halperin, Function spaces determined by a levelling length function, Can. J. Math., 5 (1953), 576-592.

3. W. A. J. Luxemburg and A. C. Zaanen, Notes on Banach function spaces, Proc. Acad. Sci., Amsterdam; Note VI, A66 (1963), 251-263; Note VII, A66 (1963), 669-681; Note VIII, $A 67$ (1964), 104-119; Note X, $A 67$ (1964), 493-506; Note XI, $A 67$ (1964), 507 518.

4. M. Morse and W. Transue, Semi-normed vector spaces with duals of integral type, J. Analyse Math., 4 (1955), 149-186.

5. - Vector subspaces of $C^{E}$ with duals of integral type, J. de Math., 37 (1958), 344-363.

6. - The existence of vector function spaces with duals of integral type, Colloq. Math., 6 (1958), 95-117.

7. H. Nakano, Semi-ordered linear spaces (Tokyo, 1955) (or Uber Erweiterungen von allgemein teilweisegeordneten Moduln, II, Prod. Imp. Acad. Tokyo, 19 (1943), 138-143).

California Institute of Technology,

Pasadena, California, and

Queen's University,

Kingston, Ontario 